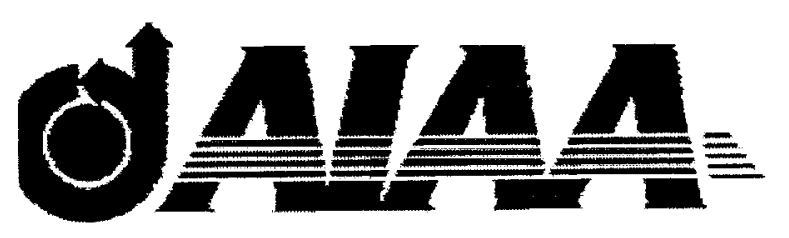

AIAA 2002-0539

Error-Based

Design Space Windowing

Melih Papila, Nilay U. Papila, Wei Shyy,

Raphael T. Haftka and Norman Fitz-Coy

Department of Aerospace Engineering, Mechanics

\& Engineering Science

University of Florida, Gainesville, FL 


\title{
ERROR-BASED DESIGN SPACE WINDOWING
}

\author{
Melih Papila* (papila@aero.ufl.edu) Nilay U. Papila* (nilay@aero.ufl.edu) \\ Wei Shyy ${ }^{\dagger}$ (wss@aero.ufl.edu) Raphael T. Haftka ${ }^{\dagger}$ (haftka@ufl.cdu) \\ Norman Fitz-Coy (nfc@aero.ufl.edu)
}

\author{
Department of Aerospace Engineering, Mechanics \& Engineering Science \\ University of Florida, Gainesville, FL 32611
}

\begin{abstract}
Windowing of design space is considered in order to reduce the bias errors due to low-order polynomial response surfaces (RS). Standard design space windowing (DSW) uses a region of interest by setting a requirement on response level and checks it by a global RS predictions over the design space. This approach, however, is vulnerable since RS modeling errors may lead to the wrong region to zoom on. The approach is modified by introducing an eigenvalue error measure based on point-topoint mean squared error criterion. Two examples are presented to demonstrate the benefit of the error-based DSW.
\end{abstract}

\section{INTRODUCTION}

The popularity of response surface (RS) techniques in design optimization studies has brought attention to ways of increasing the accuracy of RS approximations. Adequacy and accuracy of RS are mainly affected by the following three factors:

- Use of finite number of data points due to cost of data generation

- $\quad$ Noise in the data

- Inadequacy of the fitting model

We are mainly focused in this paper on model inadequacy or bias error due to use of low-order polynomials such as quadratic RS.

An obvious way for reducing bias error is to use higher order polynomials or more complex functions in RS. Cubic or even higher order polynomials were applied, for instance, by Venter et al. [1] and by Papila $(\mathrm{N})$ et al. [2].

Copyright $\bigcirc 2000$ by authors. Published by the American Institute of Aeronautics and Astronautics, Inc. with permission.

" Student Member AIAA

${ }^{\dagger}$ Fellow AIAA

${ }^{\S}$ Member AIAA
Papila and Haftka [3-4] also achieved substantial improvement in accuracy by using cubic polynomials in RS approximation for HSCT wing bending material weight.

A high-order fitting model, however, requires a large number of data points, which is usually prohibitive in high dimensional problems.

Design of experiments (DOE) offers minimum-bias criterion for reducing modeling (bias) error when loworder models are used. Venter and Haftka [5] developed an algorithm implementing minimum-bias based criterion, necessary for an irregularly shaped design space where no closed form solution exist for minimum-bias design.

Decreasing bias error is also possible by reducing the size of the fitting region by the use of reasonable-designspace (RDS) approach. The approach starts by identifying constraints specific to the problem of interest provided that they are easy and inexpensive to evaluate. These constraints are then used to eliminate unreasonable designs from the original design space. For instance, simple geometric constraints where applicable may prevent combinations of design variables resulting in unreasonable geometry configurations $[4,6-8]$. Finally, tools of DOE such as D-optimality select data points within the region of interest where response is evaluated.

It is also possible to identify region or regions of interest by windowing the design space simply based on the observed or predicted response levels over the design space [2]. The windowing approach shares the sprit of RDS approach in that it reduces bias errors by reducing the size of the design domain. However, windowing needs data generation and a global RS beforehand unlike traditional RDS approach.

In this paper, we aim to focus on improving the RS accuracy particularly in the regions critical to design optimization. We limit ourselves to quadratic RS approximation and concentrate on effective use of design space windowing (DSW) approach relying on a global RS. Standard DSW uses a requirement set on the response level and checks it by using a global RS predictions over 
the whole design space. This approach, however, is vulnerable since poor accuracy in the global RS may lead us to zoom on the wrong region.

At this point, we modify the approach and call it as error-based DSW by introducing a bias error measure. The error measure is based on an eigenvalue problem obtained by point-wise mean squared error criterion. The eigenvalue problem was derived by Papila and Haftka [9] and used as a tool to map qualitatively the RS bias error by the associated eigenvalues. In the proposed approach, regions with high eigenvalues corresponding to potential large bias error are excluded from consideration.

The following section presents more detailed description of the mean squared error criterion and use of eigenvalues in the DSW approach. Section 3 describes example problems used to demonstrate the approach. Section 4 presents the results and discussion followed by concluding remarks in Section 5. The derivation of eigenvalue problem along with the background for RS methodology can be found in the Appendix.

\section{APPROACH}

\subsection{Mean Squared Error Criterion-Eigenvalues}

An approach for estimating RS approximation bias errors due to fitting model inadequacy is presented in Ref. [9]. The mean squared error predictor (MSEP) for an inadequate model is studied point-to-point yielding an eigenvalue problem where the maximum eigenvalue at each point provides a relative estimate of maximum bias error. With the calculation of the maximum eigenvalues over the design space, regions of possible high bias error are identified. The derivation presented in Ref. [9] can also be found in this paper as an Appendix. As can be seen from the derivation the eigenvalue error measure strongly depends on the DOE used, but not on the response data.

Papila and Haftka [9] used face-centered central composite design (FCCD) and demonstrated the use of eigenvalue estimate of bias error for problems where the true function is a cubic while the fitting model is quadratic. In particular, positive correlation between the square-root of maximum eigenvalues $\sqrt{\lambda_{G}}$ and the absolute residuals was found for the examples of $2 \mathrm{D}$ polynomials that were studied.

We also use FCCD as our original DOE, quadratic RS as our fitting model and calculate the eigenvalues as if the true function is a cubic. Figure 1 presents the FCCD points and relevant $\sqrt{\lambda_{G}}$ field.

\subsection{Design Space Windowing Approach}

We adopted two types of DSW approaches based on different reasonability conditions while windowing for the design region or regions of interest. For simplicity, we consider problems where we are interested in the high response regions.
The standard $D S W$ uses Eq. (1) as the reasonability condition.

$$
\hat{y}_{R S 1} \geq y_{\text {int erest }}
$$

where $\hat{y}_{R S 1}$ is the prediction by the global RS (RS1) and $y_{\text {int erest }}$ is the response bound to define the region of interest.

Error-based DSW modifies the standard DSW by adding another condition based on the eigenvalues [9] that characterize the modeling error. In order to set a precaution for the possible misleading information due to inaccuracy of the global RS (RS1), our reasonability conditions in error-based DSW can be written as follows.

$$
\begin{aligned}
& \hat{y}_{R S 1} \geq y_{\text {int erest }} \\
& \text { and } \\
& \sqrt{\lambda_{G}} \leq \operatorname{mean}\left(\sqrt{\lambda_{G}}\right)
\end{aligned}
$$

where mean $\left(\sqrt{\lambda_{G}}\right)$ is the mean over the design space. Design points with eigenvalues less than their mean over the design space are more likely to be accurately predicted by the global RS (RS1).

Figure 2 presents the generalized flowcharts for the standard and error-based DSW approaches. The following descriptions give the details of our implementation in the flowcharts.

\section{Standard DSW Approach (Figure 2a):}

Step I: We start with a standard DOE, face-centered central composite design (FCCD) for Data set 1 and construct a global RS approximation (RS1).

Step 2: We use the global RS (RS1) to predict the response values on design points of a fine grid netting the whole design space.

Step 3: We identify design region or regions of interest based on the predictions in Step 2. We build a pool of supposedly reasonable designs by simply disregarding the designs violating the condition in Eq. (1). This is done for each identified region in case of multiple disjoint regions of interest.

Step 4: Among the designs located in the pool of Step 3 , the number of data points is reduced to a desirable amount by using D-optimality. We include design points of Data set 1 where data itself is in the region of interest $\left(y \geq y_{\text {int erest }}\right)$. This reduces the number of additional evaluations for creating Data set 2 . We then construct new RS (RS2) approximation to be used over the associated region only. (In case of multiple regions separate RS2 s are constructed)

\section{Error-based DSW Approach (Figure 2b):}

Step 1: Same as Step I of standard approach

Step 2: We use the global RS (RS1) to predict the response values and calculate the eigenvalues on design points of a fine grid netting the whole design space. 
Step 3: We identify design region or regions of interest combining eigenvalue information $\left(\sqrt{\lambda_{G}}\right)$ with the standard DSW approach by Eq. (2).

Step 4: Same as Step 4 of standard approach except denoting RS in refined regions as RS3.

\section{TEST PROBLEMS}

We investigate two different test problems to help assess the performance of the strategies explained above: (1) Two dimensional preliminary supersonic turbine design, and (2) A quartic polynomial in two dimensions. The main reason for choosing $2 \mathrm{D}$ problems is that we want to visualize easily the response surface, prediction error and eigenvalue field distributions.

\subsection{Preliminary Supersonic Turbine Design in Two- Dimension}

In our previous efforts $[2,10-14]$, we have studied the preliminary and detailed design optimization of supersonic turbines for reusable launch vehicle (RLV). A two-variable version of the two-stage turbine design problem is considered in this study. The design variables are the mean diameter, $\mathrm{D}$, and $R P M(5.081 \leq D(i n) \leq 15.243$ and $21,977 \leq R P M \leq 40,814)$. They are normalized as coded variables $x_{1}$ and $x_{2}$ in $(-1,1)$, respectively [15]. The design domain of the coded variables is a square as shown in Figure 1.

The numerical simulations are based on the aerodynamic design software called Meanline Flow Path Generator $[10,16]$ that allows rapid analyses of turbine flow fields. Using the overall turbine and stage input, the Meanline code first generates a candidate turbine flow path and displays a plot of the elevation view. The code then runs a 1-D quick aerodynamic analysis, calculating gas conditions, velocity triangles, and required number of airfoils, predicted efficiency and power output. In turbomachinery design problems, high efficiency and low weight systems are sought. For this design problem, the compromise between these two criteria can be quantified by a single response that is payload of the RLV. Therefore, the output or response of interest from the Meanline code is the change in payload compared to a fixed baseline design (i.e. 4 pay). We are mostly interested in positive $\Delta$ pay designs (i.e., $\Delta p a y>0$ ).

\subsection{Quartic Polynomial in Two-Dimension}

We wanted to mimic the efficiency data of supersonic turbine blade shape optimization problem [10] in terms of the range of response values as varying between 0 and 1 . Since we are mainly interested in high efficiency regions in turbomachinery designs, we will consider $y>0.7$ as the region of interest for this problem.

A quartic function in 2-D is devised with variables ranging between -1 and +1 (as coded variables). The quartic polynomial ranges between 0 and 1 is given in Eq. 3

$$
\begin{aligned}
& y=0.742+0.000486 x_{1}^{2}+0.000486 x_{1} x_{2} \\
& -0.242704 x_{2}^{2}+0.012646 x_{1}^{3}+0.000486 x_{1}^{2} x_{2} \\
& +0.000486 x_{1} x_{2}^{2}+0.486381 x_{1}^{3} x_{2}
\end{aligned}
$$$$
\text { where } x_{1} \text { and } x_{2} \text { range in }(-1,+1) \text { as shown in Figure } 1 .
$$

\section{RESULTS AND DISCUSSION}

We assess the accuracy of RS using mainly root-meansquared $(r m s)$ error calculations.

- rms-error Predictor: $\sqrt{\frac{\sum_{i}^{N}\left(y_{i}-\hat{y}_{i}\right)^{2}}{N-n_{b}}}$
- Testing rms-error: $\sqrt{\frac{\sum_{i}^{M-N}\left(y_{i}-\hat{y}_{i}\right)^{2}}{M-N}}$

where $N, n_{b}, M$ number of data set points, number of coefficients in RS and number of design points in the design space grid, respectively.

-Testing rms-error in $y \geq y_{\text {int erest }}: \sqrt{\frac{\sum_{i}^{K}\left(y_{i}-\hat{y}_{i}\right)^{2}}{K}}$ where $K$ is the number of the testing data in $y \geq y_{\text {int erest }}$ region excluding the data points of the associated RS.

Face-centered central composite design (FCCD) as shown in Figure 1a is used for fitting a quadratic global RS (RS1). We use 21 by 21 grid over the design space for the evaluation and assessment of the methods are used. Therefore, $N=9, n_{b}=6$ and $M=441 . \quad K$ is problem dependent as reported on the tables.

The eigenvalue distribution $\left(\sqrt{\lambda_{G}}\right)$ is a function of DOE, the fitting model and assumed true model [9]. We use FCCD as our DOE, quadratic RS as our fitting model and calculate the eigenvalues as if the true function is a cubic. Therefore distribution of $\sqrt{\lambda_{G}}$ is identical for both problems as shown in Figure $1 \mathrm{~b}$.

\section{(i) Results for Turbine Design}

The Meanline results (exact function for turbine design problem) are shown as a contour plot in Figure 3a. Three quadratic RS models were studied. The details of these RS models are given below.

- RS 1: Quadratic RS based on 9 design selected by standard FCCD (Figure la). Its prediction error contours are given in Figure $3 \mathrm{~b}$.

- RS 2: Quadratic RS based on 9 reasonable designs using standard DSW condition. Four of the points are original FCCD points, which satisfy $\triangle p a y>0$. The other five designs are selected from the designs of 
positive RS 1 predictions of the Apay calculated in 21 by 21 grid over the design space. Design points are shown in Figure 4a.

- RS 3: Quadratic RS based on 9 reasonable designs using error-based DSW conditions. Four of them are FCCD points, which satisfy $\triangle$ pay $>0$. The other five designs are selected from the designs where RS I predicts positive 4 pay and eigenvalue condition satisfied. Design points are shown in Figure 4b. Table 1 summarizes the statistics of the three RS of the turbine problem. It appears that $r m s$-error predictor of RS1 is conservative as the testing rms-error is less than the half of the predictor. Testing rms-error in region of interest is 63.5 , slightly higher than overall testing rms-error. Both approximations of windowing approaches, RS2 and RS3, resulted in smaller testing rms-error in region of interest than RSI as expected. There is factor of about 2.8 and 4.2 in the magnitudes for RS2 and RS3, respectively, compared to RS1. This indicates that error-based DSW approach improved on the standard one. The substantial increase in the overall testing rms-error and max. error reflects the fact DSW approach approximations RS2 and RS3 are not accurate outside of the region of interest. This can also be observed on the RS2 and RS3 prediction error contours, Figure $5 \mathrm{a}$ and Figure $5 \mathrm{~b}$, respectively. Comparison of error contours given in Figure $3 \mathrm{~b}$ and Figure 5 demonstrate the benefit from the windowing approaches where we are most interested. In spite of the fact that maximum error in region of interest by RS3 is higher than RS2 (Table 1), more uniform lowerror distribution in Figure $5 \mathrm{~b}$ compared to Figure $5 \mathrm{a}$ and reduction in testing rms-error in the same region shows us that we benefit from eigenvalue information during windowing.

In order to check if the eigenvalues (bias error measure) helped us to select design points with more accurate RS1 predictions, we compare error at selected points in Table 2 and Table 3. The tables report the error measure $\sqrt{\lambda_{G}}$ and RS1 prediction errors at the RS2 and RS3 design points, respectively. Excluding four common design points coming from the original FCCD, average error (average error of the boldface rows) decreases from 66.4 in Table 2 to 45.7 in Table 3. This confirms the usefulness of the eigenvalues for this example.

\section{(ii) Results for Quartic Polvnomial}

Contour plot for quartic example response given in Figure $6 a$ shows that we can consider two separate regions of interest: upper-right and lower-left quadrants of the square design domain. This observation and the logic behind the windowing procedure lead us to evaluate each region separately. The regions, on the other hand, are not completely remote and disjoint. Therefore, we also want to see the effect of employing the DSW approaches on the connected regions as a single region of interest around one diagonal of the domain. We first present the single continuous region consideration; windowing as single region. Then present results of windowing as multiple regions.

(a) Windowing as single region

Four quadratic RS models were studied for the overall design domain. The details of these RS models are given below.

- RS 1: Quadratic RS based on 9 designs selected by standard FCCD (Figure 1). Its prediction error contours are given in Figure $6 \mathrm{~b}$

- RS 2: Quadratic RS based on 9 reasonable designs using standard DSW condition. Four of the data points are FCCD points, which satisfy $y>0.7$. The other five designs are selected from the designs of positive RS 1 predictions of the $y$ calculated in 21 by 21 grid over the design space. Design points and error contours are shown in Figure $7 \mathrm{a}$ and Figure 8a, respectively.

- RS 3: Quadratic RS based on 9 reasonable designs using error-based DSW conditions. Four of the data points are FCCD points, which satisfy $y>0.7$. The other five designs are selected from the designs where RS 1 predicts $y$ higher than 0.7 and eigenvalue condition satisfied. Design points and error contours are shown in Figure $7 \mathrm{~b}$ and Figure 8b, respesctively.

- RS 4: Quadratic RS based on RS 1 and RS 3 design points (13 designs in total). Design points are shown in Figure 9a.

Table 4 summarizes the statistics of the four RS. Unlike turbine problem, rms-error predictor of RSI (equal to zero) suggesting perfect fit gives a sense of security that is proved to be wrong by the nonzero testing $m s$-error. Testing rms-error in region of interest is even higher and equal to 0.051 . Both approximations of DSW approaches, RS2 and RS3, resulted in higher testing rms-error in region of interest than RS1. They are 0.073 and 0.063 , respectively. This indicates that error-based DSW approach improved on the standard one for the single windowing, but neither DSW helped to increase accuracy compared to RS1 with single windowing. Figure $8 \mathrm{a}$ and $\mathrm{b}$ also compares visually the two DSW approaches in terms of error distribution. It shows that error-based DSW resulted smaller errors at remote locations of the quadrants forming the region of interest.

Table 5 and Table 6 show error measure $\sqrt{\lambda_{G}}$ and RS1 prediction errors at the RS2 and RS3 design points, respectively. Excluding five common design points coming from the original FCCD average епror (average error of the boldface rows) decreases from 0.0916 in Table 2 to 0.0868 in Table 3.

The best performance among the four RS was obtained by RS4 (Figure 9). Although its rms-error predictor is the largest we obtained smallest overall testing rms-error, 
testing rms-error and maximum error in the region of interest. In other words, adding new data points selected by DSW to the original FCCD improved the accuracy.

\section{(b) Windowing as multiple regions}

We report results only on the upper-right quadrant since the results of lower-left quadrant are mirror image of the upper right. For the statistics and design points we consider 11 by 11 grid points in the quadrant. Two RS were constructed as described below

- RS 5: Quadratic RS based on 9 reasonable designs using standard DSW condition. Three of them are FCCD points, which satisfy $y>0.7$. The rest is selected from the designs of RS 1 predictions exceeding 0.7. Design points for RS 5 are shown Figure 10a.

- RS 6: Quadratic RS based on 9 reasonable designs using error-based DSW condition. Three of them are FCCD points, which satisfy $y>0.7$. The rest of is selected from the designs where RS 1 predicts $y$ higher than 0.7 and eigenvalue condition satisfied. Design points for RS 5 are shown Figure 10b.

Table 7 summarizes the statistics of the two RSs. Overall testing rms-error and testing rms-error in region of interest is lower by standard DSW than error-based DSW (overall: 0.042 and 0.046 , respectively, and in the region of interest: 0.026 and 0.030 , respectively). In other words, standard DSW did a better job compared to error-based approach in this example. One possible reason is that introducing the error-based condition may increase irregularity of the domain of interest. We also calculated the RSl testing rms-error in region of interest over this particular quadrant as 0.061 that shows both DSW approaches helped us to lower by half. Figure 11 presents the error contour for RS5 and RS6. The location of high errors seemed to be shifted by the error-based DSW.

Table 8 and Table 9 present eigenvalue based error measure $\sqrt{\lambda_{G}}$ and RS1 prediction errors at the RS5 and RS6 design points, respectively. Although we did not see benefit from the error-based DSW in terms of statistics, these tables show that eigenvalues condition in fact selected points with lower errors. Excluding five common design points average error (average error of the boldface rows) decreases from 0.0943 to to 0.0658 .

\section{CONCLUDING REMARKS}

In this paper we focused on the model inadequacy or bias error due to use of low-order polynomials such as quadratic RS and increased accuracy by design space windowing (DSW). We set a requirement on the response based on a global RS and zoom with a local RS on that region. Since the modeling errors may lead us to the wrong region to zoom on, we integrated an eigenvalue error measure into the procedure and called it error-based DSW. Two examples were studied to demonstrate the benefit from error-based DSW:
- In two-dimensional two-stage turbine problem one region of interest was identified. Both DSW approaches improved on the global RS accuracy. Statistics showed that accuracy obtained after errorbased DSW increased in the region of interest compared to standard DSW

- In quartic polynomial example two regions were identified. In each region DSW approaches offered substantial improvement in accuracy compared to global RS. The error-based DSW, however, did not bring improvement over the standard approach possibly due to increased irregularity of the region of interest caused by the eigenvalue condition.

- For both examples, the average errors at the data points are lower after the selection by error-based DSW. This is an indication that modified approach employing eigenvalues disregarded design points where reasonability based on RSI prediction may be misleading.

\section{ACKNOWLEDGEMENT}

This work has been partially supported by NASA Marshall Space Flight Center, Mr. Kevin Tucker project monitor and by NASA Grant \# NAG1-2177.

\section{REFERENCES}

1. Venter, G., Haftka, R. T., and Starnes, J. J. H., "Construction of Response Surface Approximations for Design Optimization," $6^{\text {th }}$ AIAA/USAF/NASA/ISSMO Symposium on Multidisciplinary Analysis and Optimization, Bellevue, Washington, 1996.

2. Papila, N., Shyy, W., Griffin, L., Huber, F., and Tran, K., "Preliminary Design Optimization for a Supersonic Turbine for Rocket Propulsion," AIAA/SAE/ASME/ASEE 36th Joint Propulsion Conference, AIAA Paper 2000-3242, June 2000.

3. Papila, M. and Haftka, R. T., "Uncertainty and Wing Structural Weight Approximations," Proceedings, 40th AIAA/ASME/ASCE/ASC Structures, Structural Dynamics, and Material Conference, Paper AIAA-991312, pp. 988-1002, St. Louis, MO, April 1999.

4. Papila, M. and Haftka, R. T., "Response Surface Approximations: Noise, Error Repair and Modeling Errors," AIAA Journal, 38(12), pp. 2336-2343, 2000.

5. Venter, G., and Haftka R.T., "Minimum-Bias Based Experimental Design for Constructing Response Surfaces in Structural Optimization" Proceedings, the 38th AIAA/ASME/ASCE/AHS/ASC Structures, Structural Dynamics, and Materials Conference, Paper AIAA-971053, Part 2, pp. 1225-1238, Kissimmee, Florida, April 7. $10,1997$.

6. Kaufman, M., Balabanov, V., Burgee, S. L., "Variable-Complexity Response Surface Approximations for Wing Structural Weight in HSCT Design," Computational Mechanics, 18, pp. 112-126, 1996. 
7. Roux, W.J., Stander, N., and Haftka, R.T., "Response Surface Approximations for Structural Optimization," International Journal for Numerical Methods in Engineering, 42, pp. 517-534, 1998.

8. Balabanov, V. O., Giunta, A. A., Golovidov, O., Grossman, B., Mason, W.H., Watson, L.T., and Haftka, R.T., "Reasonable Design Space Approach to Response Surface Approximation," Joumal of Aircraft Vol. 36, No.1, 1999, pp. 308-315.

9. Papila, M. and Haftka, R. T., "Uncertainty and Response Surface Approximations," 42nd AIAA/ASME/ASCE/ASC Structures, Structural Dynamics, and Material Conference, Paper AIAA-011680, Seattle, WA, April 2001.

10. Papila, N., Shyy, W., Griffin, L., and Dorney, D.J., "Shape Optimization of Supersonic Turbines Using Response Surface and Neural Network Methods," AIAA 39th Aerospace Sciences Meeting \& Exhibit, Reno, Neveda, AIAA Paper 2001-1065, January 2001.

11. Shyy, W., Papila, N., Tucker, P. K., Vaidyanathan, R., and Griffin L., "Global Design Optimization for Fluid Machinery Applications," Proceeding of the Second International Symposium on Fluid Machinery and Fluid Engineering, October, Beijing, China, 2000, pp.1-10.

12. Shyy, W., Papila, N., Vaidyanathan, R., and Tucker, P.K., "Global Design Optimization for Aerodynamics and Rocket Propulsion Components," Progress in Aerospace Sciences, Vol. 37, 2001, pp.59-118.

13. Vaidyanathan, R., Papila, N., Shyy, W., Tucker, P.K., Griffin, L. W., Fitz-Coy, N., and Haftka, R.T, "Neural Network-based and Response Surface-based
Optimization Strategies for Rocket Engine Component Design," 8th AIAA/USAF/NASA/ISSMO Symposium on Multidisciplinary Analysis and Optimization, Long Beach, Califomia, September 2000.

14. Griffin, L. W., Dorney, D. J., Huber, F., Shyy, W., Papila, N., and Tran, K., "Detailed Aerodynamic Design Optimization of an RLV Turbine," AIAA/SAE/ASME/ASEE 37th Joint Propulsion Conference and Exhibit, Salt Lake City, Utah, AIAA Paper 2001-3397, June 2001.

15. Myers, R. H., and Montgomery, D. C., Response Surface Methodology -Process and Product Optimization Using Designed Experiments, New York: John Wiley \& Sons, Inc., 1995, pp.208-279.

16. Huber, Frank, "Turbine Aerodynamic Design Tool Development", presented at the Space Transportation Fluids Workshop, Marshall Space Flight Center, AL, April 2001 .

17. Haftka, R.T., Scott, E.P., and Cruz, J.R., "Optimization and Experiments: A Survey," Applied Mechanics Reviews, Vol. 51, No. 7, 1998, pp. 435-448.

18. Khuri, A. I. and Cornell, J. A., Response Surfaces: Designs and Analyses, 2nd edition, New York, Marcel Dekker Inc., 1996, pp. 207-247.

19. Seber, G. A. F., Linear Regression Analysis, New York, Wiley, 1977.

Table 1: Statistics of the global fits for turbine design problem

\begin{tabular}{|l|c|c|c|}
\hline & $\begin{array}{c}\text { RS 1 } \\
\text { (FCCD) }\end{array}$ & $\begin{array}{c}\text { RS 2 } \\
\text { (Standard DSW) }\end{array}$ & $\begin{array}{c}\text { RS 3 } \\
\text { (Error-based DSW) }\end{array}$ \\
\hline RSquare Adj & 0.984 & 0.998 & 0.998 \\
\hline rms-error Predictor & 151.947 & 19.053 & 22.103 \\
\hline Mean & -432.831 & 513.476 & 502.470 \\
\hline Observations in $\Delta$ pay $>0 /$ Observations, $N$ & $4 / 9$ & $9 / 9$ & $9 / 9$ \\
\hline Testing rms-error & 60.583 & 171.830 & 133.616 \\
\hline \#of the testing data, $(M-N)$ & 432 & 432 & 1532 \\
\hline Testing rms-error in $\Delta$ pay $>0$ & 63.546 & 22.645 & 188 \\
\hline \#of the testing data in $\Delta$ pay $>0, K$ & 193 & 188 & 554.938 \\
\hline Mean of the testing data in $\Delta$ pay $>0$ & 505.870 & 563.886 & 96.660 \\
\hline Max. Error in $\Delta$ pay $>0$ & 160.897 & 45.356 & 692.394 \\
\hline Max. Error & 160.897 & 885.188 & 106 \\
\hline
\end{tabular}


Table 2: RS1 error at RS 2 data points for turbine design problem. Bold face rows are design points selected by Standard DSW. Average error of the boldface rows is 66.4

\begin{tabular}{|c|c|c|c|c|c|}
\hline D & RPM & $\Delta$ pay & $\left(\hat{\Delta}_{p a y}\right)_{R S I}$ & $\sqrt{\lambda_{G}}$ & Error \\
\hline $\mathbf{- 0 . 5}$ & $\mathbf{1}$ & $\mathbf{2 1 2 . 6 6}$ & $\mathbf{6 2 . 5 1}$ & $\mathbf{0 . 7 1 5}$ & $\mathbf{1 5 0 . 1 5}$ \\
\hline 0 & 0 & 15.75 & 4.58 & 0.497 & 11.17 \\
\hline 0 & 1 & 806.18 & 677.31 & 0.832 & 128.87 \\
\hline $\mathbf{0 . 3}$ & $\mathbf{1}$ & $\mathbf{9 8 3 . 0 4}$ & $\mathbf{9 2 8 . 9 0}$ & $\mathbf{0 . 7 9 2}$ & $\mathbf{5 4 . 1 4}$ \\
\hline $\mathbf{0 . 6}$ & $\mathbf{- 0 . 5}$ & $\mathbf{1 8 . 7 1}$ & $\mathbf{1 6 . 2 2}$ & $\mathbf{0 . 6 5 4}$ & $\mathbf{2 . 4 9}$ \\
\hline $\mathbf{1}$ & $\mathbf{- 0 . 6}$ & $\mathbf{1 3 0 . 7 7}$ & $\mathbf{4 0 . 5 1}$ & $\mathbf{0 . 6 6 2}$ & $\mathbf{9 0 . 2 6}$ \\
\hline 1 & 0 & 630.5 & 565.97 & 0.832 & 64.53 \\
\hline $\mathbf{1}$ & $\mathbf{0 . 2}$ & $\mathbf{7 4 9 . 2 9}$ & $\mathbf{7 1 4 . 3 4}$ & $\mathbf{0 . 8 1 5}$ & $\mathbf{3 4 . 9 5}$ \\
\hline 1 & $\mathbf{1}$ & 1074.38 & 1173.87 & 0.762 & 99.49 \\
\hline
\end{tabular}

Table 3: RS1 error at RS 3 data points for turbine design problem. Bold face rows are design points selected by error-based DSW. Average error of the boldface rows decreases to 45.7

\begin{tabular}{|c|c|c|c|c|c|}
\hline $\mathbf{D}$ & RPM & $\Delta$ pay & $\left(\hat{\Delta}_{p a y}\right)_{R S 1}$ & $\sqrt{\lambda_{G}}$ & Error \\
\hline $\mathbf{- 0 . 3}$ & $\mathbf{0 . 5}$ & $\mathbf{1 0 8 . 4 1}$ & $\mathbf{3 3 . 5 2}$ & $\mathbf{0 . 6 3 4}$ & $\mathbf{7 4 . 8 9}$ \\
\hline 0 & 0 & 15.75 & 4.58 & 0.497 & 11.17 \\
\hline 0 & 1 & 806.18 & 677.31 & 0.832 & 128.87 \\
\hline $\mathbf{0 . 3}$ & $\mathbf{- 0 . 3}$ & $\mathbf{4 . 6}$ & $\mathbf{1 4 . 3 4}$ & $\mathbf{0 . 5 6 8}$ & $\mathbf{9 . 7 4}$ \\
\hline $\mathbf{0 . 5}$ & $\mathbf{0 . 4}$ & $\mathbf{7 2 8 . 8 9}$ & $\mathbf{7 0 3 . 7 6}$ & $\mathbf{0 . 6 4 5}$ & $\mathbf{2 5 . 1 3}$ \\
\hline $\mathbf{0 . 7}$ & $\mathbf{1}$ & $\mathbf{1 0 6 8 . 9 1}$ & $\mathbf{1 1 2 7 . 5 3}$ & $\mathbf{0 . 6 1 3}$ & $\mathbf{5 8 . 6 2}$ \\
\hline $\mathbf{0 . 9}$ & $\mathbf{- 0 . 6}$ & $\mathbf{8 4 . 6 1}$ & $\mathbf{2 4 . 4 6}$ & $\mathbf{0 . 5 9 9}$ & $\mathbf{6 0 . 1 5}$ \\
\hline 1 & 0 & 630.5 & 565.97 & 0.832 & 64.53 \\
\hline 1 & 1 & 1074.38 & 1173.87 & 0.762 & 99.49 \\
\hline
\end{tabular}

Table 4: Statistics of the RS fits for quartic polynomial

\begin{tabular}{|l|c|c|c|c|}
\hline & $\begin{array}{c}\text { RS 1 } \\
(\text { FCCD })\end{array}$ & $\begin{array}{c}\text { RS 2 } \\
\text { (Standard } \\
\text { DSW) }\end{array}$ & $\begin{array}{c}\text { RS 3 } \\
\text { (Error-based } \\
\text { DSW) }\end{array}$ & $\begin{array}{c}\text { RS 4 } \\
\text { (RS 1 + RS 3 } \\
\text { design points })\end{array}$ \\
\hline RSquare Adj & 1.000 & 0.990 & 0.847 & 0.880 \\
\hline rms-error Predictor & 0.000 & 0.015 & 0.056 & 0.115 \\
\hline Mean & 0.5800 & 75164 & 0.829 & 0.653 \\
\hline Observations in y $>0.7 /$ Observations, $N$ & $5 / 9$ & $7 / 9$ & $7 / 9$ & $7 / 9$ \\
\hline Testing rms-error & 0.08 & 0.260 & 0.259 & 0.075 \\
\hline \#of the testing data, $(M-N)$ & 432 & 432 & 432 & 428 \\
\hline Testing $r m s-$ error in y $>0.7$ & 0.051 & 0.073 & 0.063 & 0.039 \\
\hline \#of the testing data in y $>0.7, K$ & 200 & 198 & 198 & 198 \\
\hline Mean of the testing data in $y>0.7$ & 0.789 & 0.794 & 0.765 & 0.776 \\
\hline Max Error in $y>0.7$ & 0.161 & 0.149 & 0.159 & 0.109 \\
\hline Max. Error & 0.192 & 0.778 & 0.750 & 0.217 \\
\hline
\end{tabular}


Table 5: RS 1 error at RS 2 design points for quartic polynomial. Bold face rows are design points selected by Standard DSW. Average error of the boldface rows is 0.0916

\begin{tabular}{|c|c|c|c|c|c|}
\hline $\mathbf{x}_{1}$ & $\mathbf{x}_{2}$ & $\mathbf{y}$ & $\hat{y}_{R S} 1$ & $\sqrt{\lambda_{G}}$ & Error \\
\hline-1 & -1 & 0.9728 & 0.9731 & 0.762 & 0.0003 \\
\hline-1 & 0 & 0.7296 & 0.7292 & 0.831 & 0.0003 \\
\hline$-\mathbf{0 . 5}$ & -1 & $\mathbf{0 . 5 5 8 2}$ & $\mathbf{0 . 7 3 5 8}$ & $\mathbf{0 . 7 1 5}$ & $\mathbf{0 . 1 7 7 5}$ \\
\hline 0 & 0 & 0.7417 & 0.7417 & 0.497 & 0.0000 \\
\hline $\mathbf{0 . 1}$ & $\mathbf{0}$ & $\mathbf{0 . 7 4 1 7}$ & $\mathbf{0 . 7 4 3 0}$ & $\mathbf{0 . 4 9 9}$ & $\mathbf{0 . 0 0 1 3}$ \\
\hline $\mathbf{0 . 5}$ & $\mathbf{1}$ & $\mathbf{0 . 5 6 2 1}$ & $\mathbf{0 . 7 4 9 4}$ & $\mathbf{0 . 7 1 5}$ & $\mathbf{0 . 1 8 7 3}$ \\
\hline $\mathbf{1}$ & $\mathbf{- 0 . 1}$ & $\mathbf{0 . 7 0 3 7}$ & $\mathbf{0 . 7 0 4 0}$ & $\mathbf{0 . 8 2 7}$ & $\mathbf{0 . 0 0 0 3}$ \\
\hline 1 & 0 & 0.7549 & 0.7552 & 0.831 & 0.0003 \\
\hline 1 & 1 & 1.0000 & 0.9997 & 0.762 & 0.0003 \\
\hline
\end{tabular}

Table 6: RS 1 error at RS 3 design points for quartic polynomial. . Bold face rows are design points selected by error-based DSW. Average error of the boldface rows is $\mathbf{0 . 0 8 6 8}$

\begin{tabular}{|c|c|c|c|c|c|}
\hline & & $y$ & $\hat{y}_{R S 1}$ & $\sqrt{\lambda_{G}}$ & Error \\
\hline-1 & -1 & 0.9728 & 0.9731 & 0.762 & 0.0003 \\
\hline-1 & $-\mathbf{0 . 7}$ & $\mathbf{0 . 9 5 0 9}$ & $\mathbf{0 . 9 5 0 9}$ & $\mathbf{0 . 6 1 3}$ & $\mathbf{0 . 0 0 0 0}$ \\
\hline-1 & 0 & 0.7296 & 0.7292 & 0.831 & 0.0003 \\
\hline$-\mathbf{0 . 7}$ & -1 & $\mathbf{0 . 6 6 1 5}$ & $\mathbf{0 . 8 3 0 7}$ & $\mathbf{0 . 6 1 3}$ & $\mathbf{0 . 1 6 9 2}$ \\
\hline 0 & 0 & 0.7417 & 0.7417 & 0.497 & 0.0000 \\
\hline $\mathbf{0 . 7}$ & $\mathbf{1}$ & $\mathbf{0 . 6 7 1 4}$ & $\mathbf{0 . 8 4 9 5}$ & $\mathbf{0 . 6 1 3}$ & $\mathbf{0 . 1 7 8 1}$ \\
\hline 1 & 0 & 0.7549 & 0.7552 & 0.831 & 0.0003 \\
\hline 1 & $\mathbf{0 . 7}$ & $\mathbf{0 . 9 7 7 3}$ & $\mathbf{0 . 9 7 7 3}$ & $\mathbf{0 . 6 1 3}$ & $\mathbf{0 . 0 0 0 0}$ \\
\hline 1 & 1 & 1.0000 & 0.9997 & 0.762 & 0.0003 \\
\hline
\end{tabular}

Table 7: Statistics of the RS fits for quartic polynomial in upper-right quadrant where testing $r m s$-error in $y>0.7$ is 0.061 by RS 1

\begin{tabular}{|l|c|c|}
\hline & $\begin{array}{c}\text { RS 5 } \\
\text { (Standard DSW) }\end{array}$ & $\begin{array}{c}\text { RS 6 } \\
\text { (Error-based DSW) }\end{array}$ \\
\hline RSquare Adj & 0.905 & 0.840 \\
\hline rms-error Predictor & 0.043 & 0.049 \\
\hline Mean & 0.755 & 0.778 \\
\hline Observations in y $>0.7 /$ Observations, $N$ & $7 / 9$ & $7 / 9$ \\
\hline Testing rms-error & 0.042 & 0.046 \\
\hline \#of the testing data, $(M-N)$ & 112 & 112 \\
\hline Testing rms-error in $\mathrm{y}>\mathbf{0 . 7}$ & 0.026 & 0.030 \\
\hline \#of the testing data in $\mathrm{y}>0.7, K$ & 74 & 74 \\
\hline Mean of the testing data in $\mathrm{y}>0.7$ & 0.790 & 0.796 \\
\hline Max Error in $\mathrm{y}>0.7$ & 0.060 & 0.054 \\
\hline
\end{tabular}

For the comparison, test points are the designs of the relevant quadrant. 
Table 8: RS 1 error at RS 5 design points for quartic polynomial in upper-right quadrant. Bold face rows selected by Standard DSW. Average error of the boldface rows is 0.0943

\begin{tabular}{|c|c|c|c|c|c|}
\hline $\mathbf{x}_{\mathbf{1}}$ & $\mathbf{x}_{\mathbf{2}}$ & $\mathbf{y}$ & $\sqrt{\lambda_{G}}$ & $\mathbf{R S 1}$ & Error \\
\hline 0 & 0 & 0.7417 & 0.4969 & 0.742 & 0.0000 \\
\hline 0 & 0.4 & 0.7029 & 0.5708 & 0.703 & 0.0001 \\
\hline $\mathbf{0 . 3}$ & $\mathbf{0 . 8}$ & $\mathbf{0 . 5 9 7 5}$ & $\mathbf{0 . 7 0 4 2}$ & $\mathbf{0 . 7 0 7}$ & $\mathbf{0 . 1 0 9 9}$ \\
\hline 0.5 & 0 & 0.7434 & 0.6175 & 0.748 & 0.0049 \\
\hline $\mathbf{0 . 5}$ & $\mathbf{1}$ & $\mathbf{0 . 5 6 2 1}$ & $\mathbf{0 . 7 1 4 8}$ & $\mathbf{0 . 7 4 9}$ & $\mathbf{0 . 1 8 7 3}$ \\
\hline $\mathbf{0 . 6}$ & $\mathbf{0 . 4}$ & $\mathbf{0 . 7 4 8 1}$ & $\mathbf{0 . 6 6 1 5}$ & $\mathbf{0 . 8 2 8}$ & $\mathbf{0 . 0 7 9 8}$ \\
\hline 1 & 0 & 0.7549 & 0.8315 & 0.755 & 0.0003 \\
\hline 1 & $\mathbf{0 . 5}$ & $\mathbf{0 . 9 3 8 0}$ & $\mathbf{0 . 7 1 4 8}$ & $\mathbf{0 . 9 3 8}$ & $\mathbf{0 . 0 0 0 1}$ \\
\hline 1 & 1 & 1.0000 & 0.7617 & 1.000 & 0.0003 \\
\hline
\end{tabular}

Table 9: RS 1 error at RS 6 design points for quartic polynomial in upper-right quadrant. Bold face rows selected by error-based DSW. Average error of the boldface rows is 0.0658

\begin{tabular}{|c|c|c|c|c|c|}
\hline $\mathbf{x}_{\mathbf{1}}$ & $\mathrm{x}_{\mathbf{2}}$ & $\mathbf{y}$ & $\sqrt{\lambda_{G}}$ & $\mathbf{R S 1}$ & Error \\
\hline 0 & 0 & 0.7417 & 0.4969 & 0.742 & 0.0000 \\
\hline 0 & 0.4 & 0.7029 & 0.5708 & 0.703 & 0.0001 \\
\hline $\mathbf{0 . 1}$ & $\mathbf{0 . 5}$ & $\mathbf{0 . 6 8 1 4}$ & $\mathbf{0 . 6 1 9 3}$ & $\mathbf{0 . 7 0 7}$ & $\mathbf{0 . 0 2 5 5}$ \\
\hline 0.5 & 0 & 0.7434 & 0.6175 & 0.748 & 0.0049 \\
\hline $\mathbf{0 . 5}$ & $\mathbf{0 . 3}$ & $\mathbf{0 . 7 4 0 0}$ & $\mathbf{0 . 6 3 4 1}$ & $\mathbf{0 . 8 0 0}$ & $\mathbf{0 . 0 5 9 7}$ \\
\hline $\mathbf{0 . 7}$ & $\mathbf{1}$ & $\mathbf{0 . 6 7 1 4}$ & $\mathbf{0 . 6 1 2 8}$ & $\mathbf{0 . 8 4 9}$ & $\mathbf{0 . 1 7 8 1}$ \\
\hline 1 & 0 & 0.7549 & 0.8315 & 0.755 & 0.0003 \\
\hline $\mathbf{1}$ & $\mathbf{0 . 7}$ & $\mathbf{0 . 9 7 7 3}$ & $\mathbf{0 . 6 1 2 8}$ & $\mathbf{0 . 9 7 7}$ & $\mathbf{0 . 0 0 0 0}$ \\
\hline 1 & 1 & 1.0000 & 0.7617 & 1.000 & 0.0003 \\
\hline
\end{tabular}

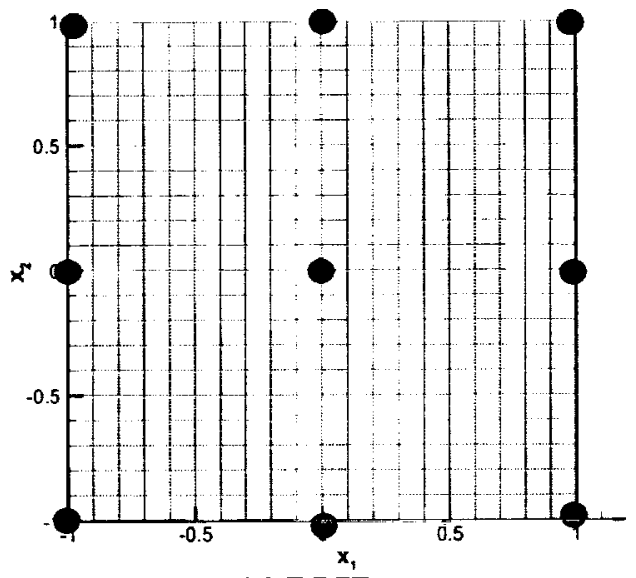

(a) FCCD

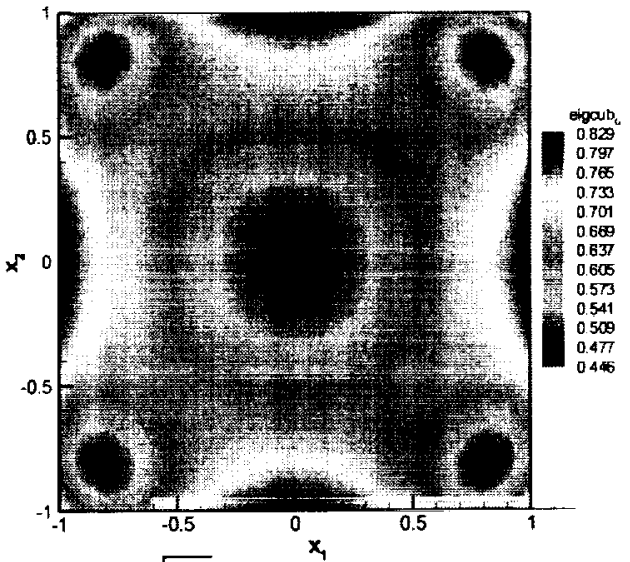

(b) $\sqrt{\lambda_{G}}$ con tours

Figure 1: Design space for two-variable examples: FCCD design points and $\sqrt{\lambda_{G}}$ con tours (Mean $\sqrt{\lambda_{G}}=0.0646$ ) 
AIAA 2002-0539

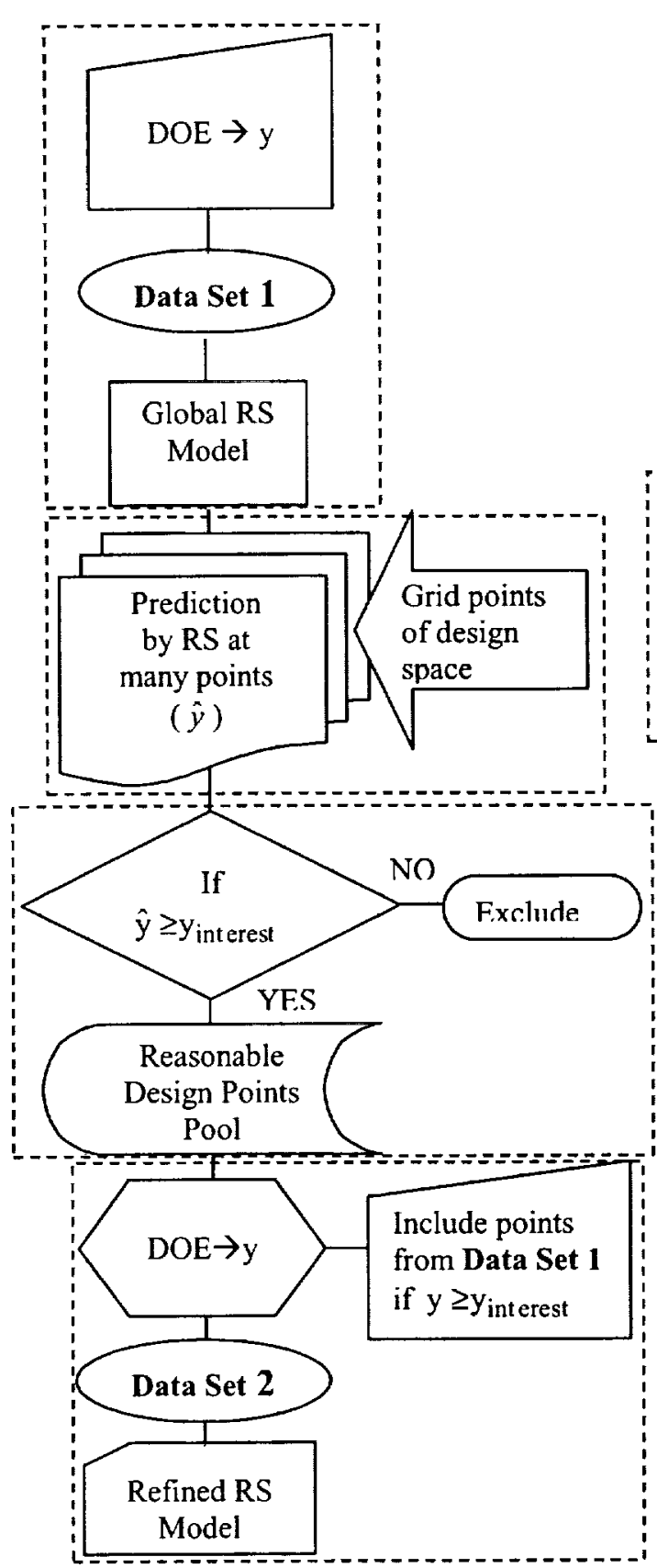

(a) Standard DSW approach

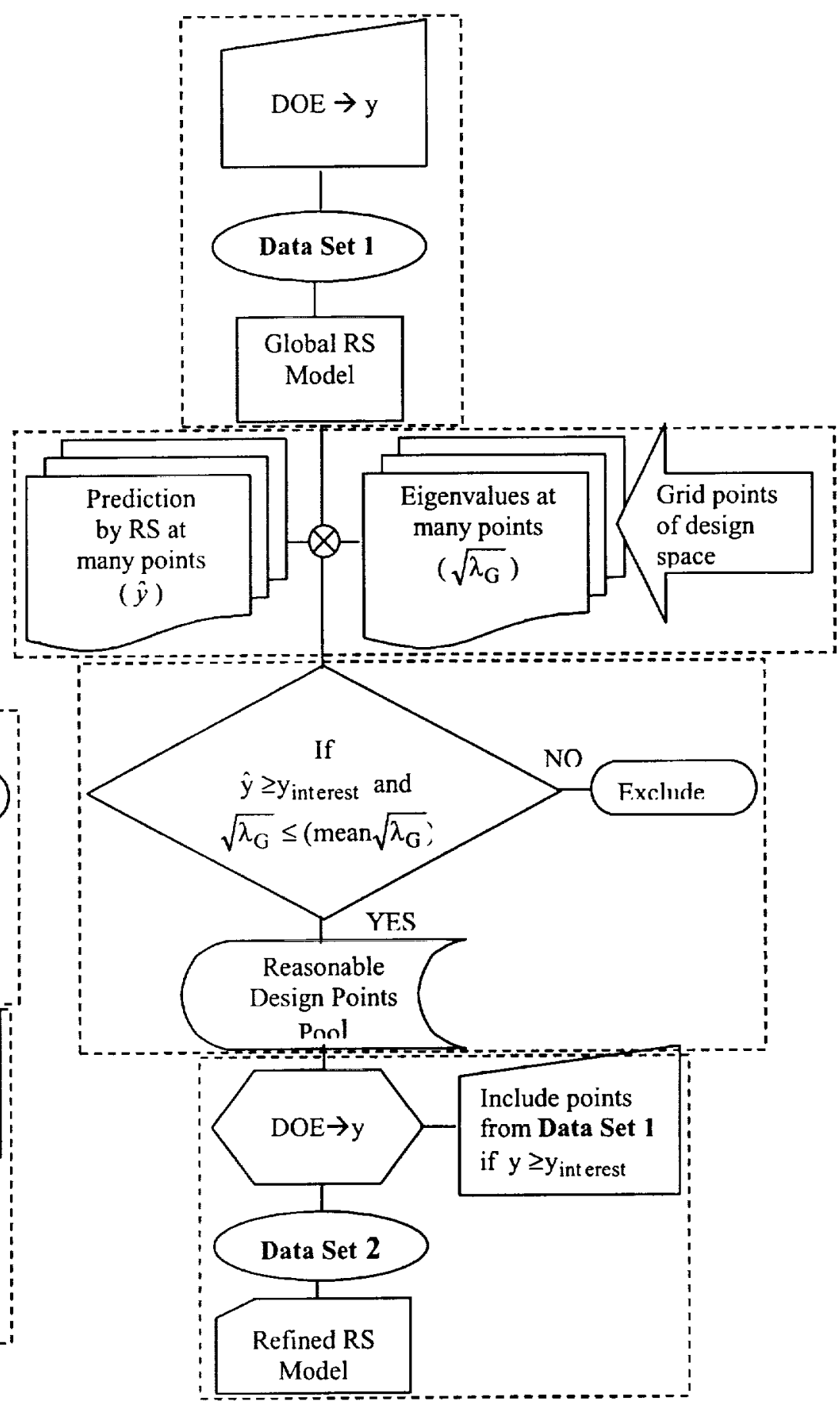

(b) Error-based DSW approach

Figure 2: Standard and error-based design space windowing approach for searching regions of high response designs 


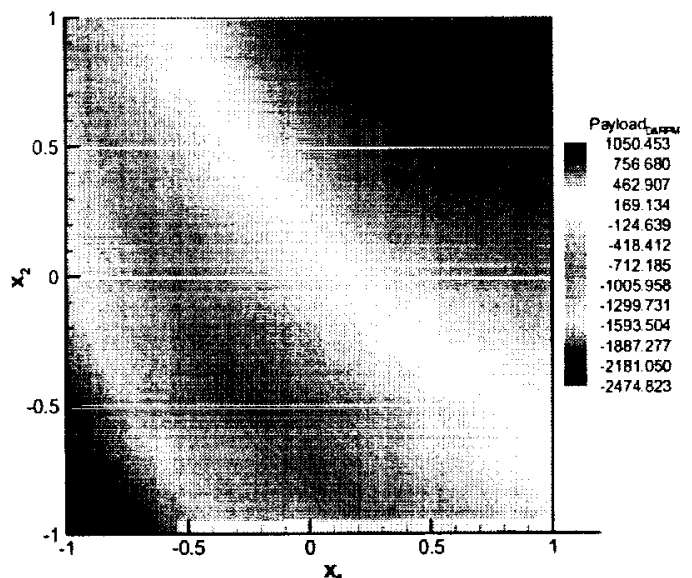

(a) True function

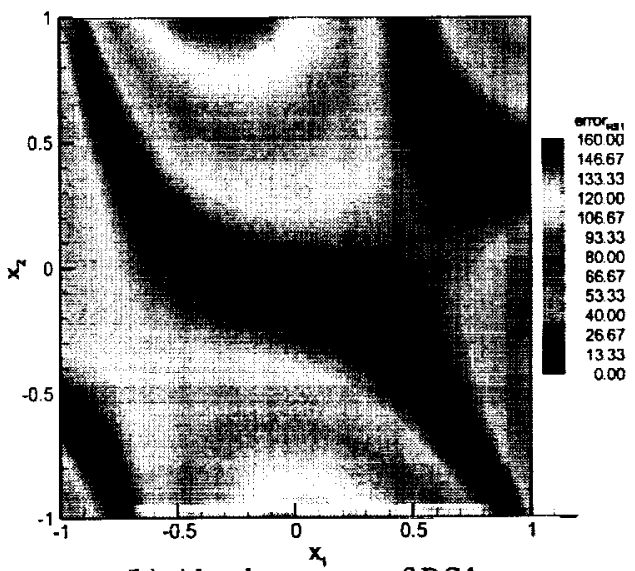

(b) Absolute error of RS1

Figure 3: $\triangle$ pay contours and RSI errors for turbine design problem $\left(x_{1}: D\right.$ and $\left.x_{2}: R P M\right)$

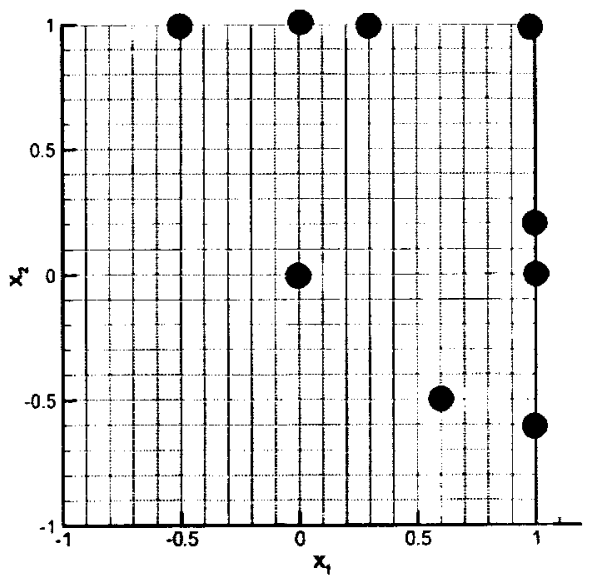

(a) RS 2 (Standard DSW)

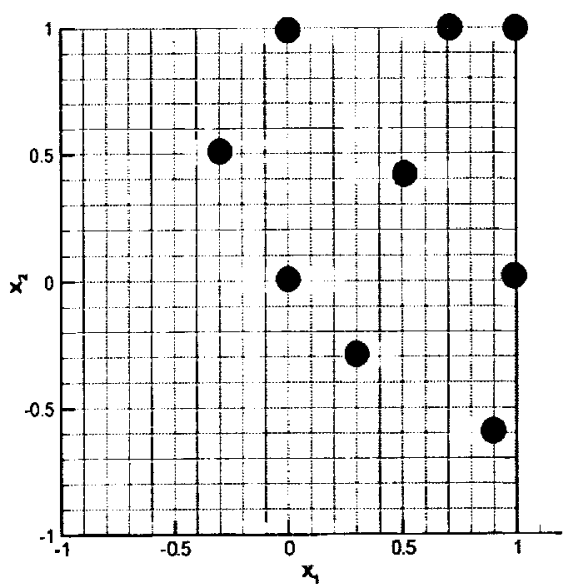

(b) RS 3 (Error-based DSW)

Figure 4: Design space for RS models for turbine design problem $\left(x_{1}: D\right.$ and $\left.x_{2}: R P M\right)$

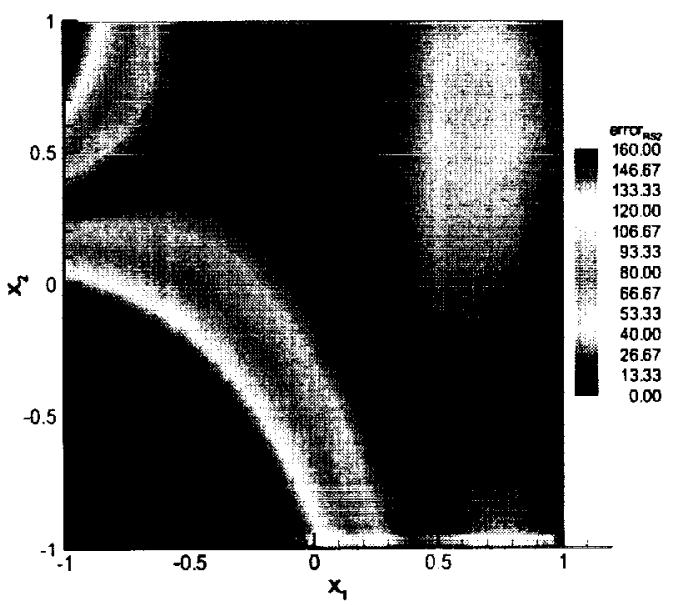

(a) RS 2 (Standard DSW)

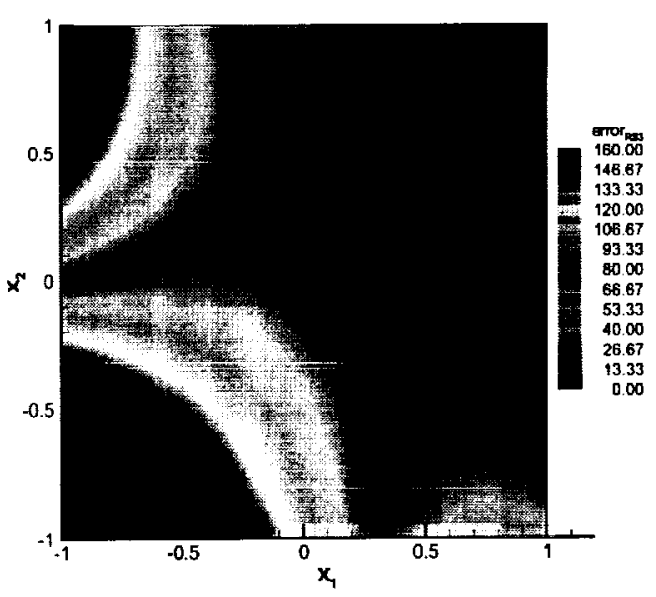

(b) RS 3 (Error-based DSW)

Figure 5: Error contours after DSW in turbine design problem $\left(x_{1}: D\right.$ and $\left.x_{2}: R P M\right)$ 


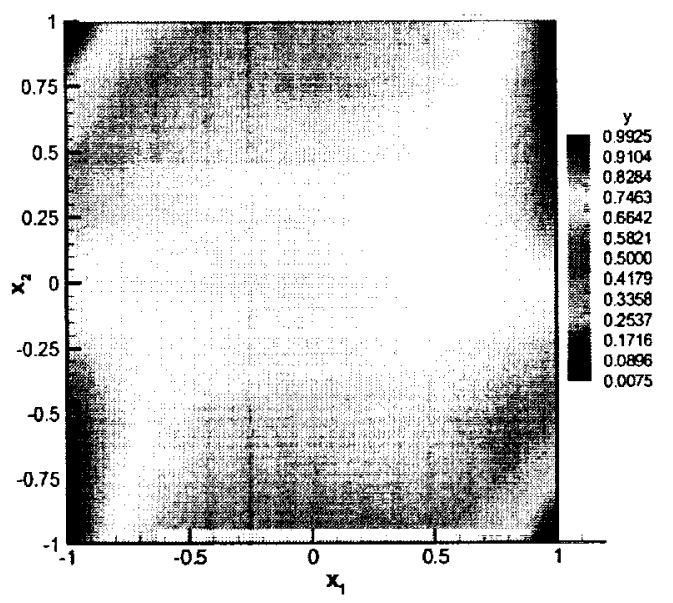

(a) True function

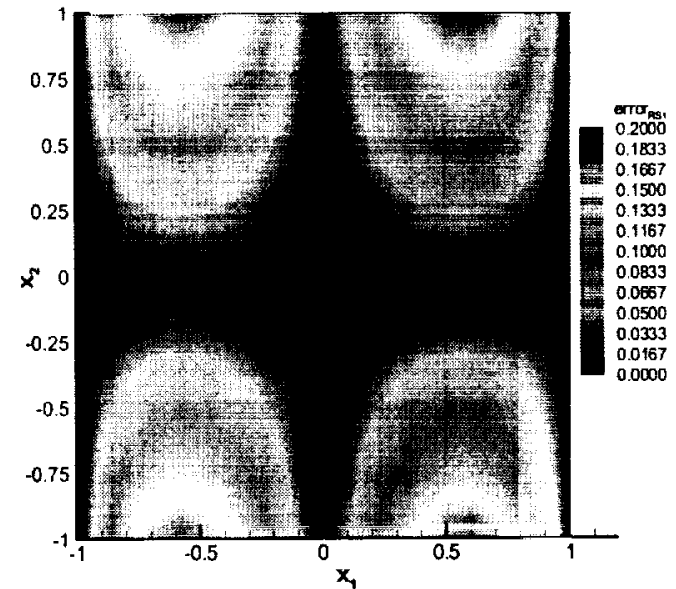

(b) Absolute error of RS1

Figure 6: Contours for y and RS1 errors in quartic polynomial

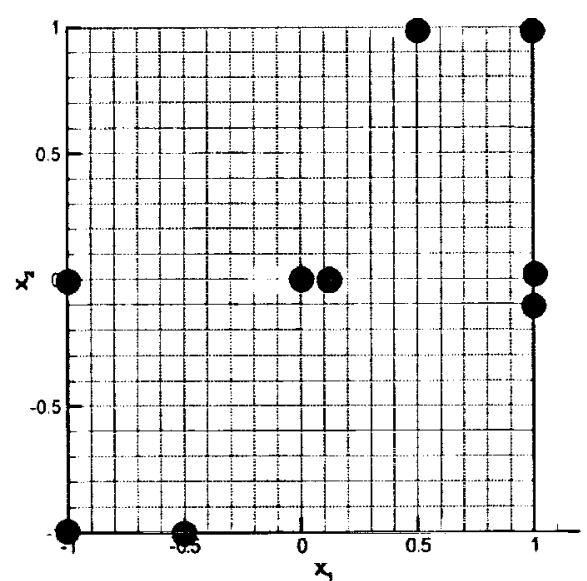

(a) RS 2 (Standard DSW)

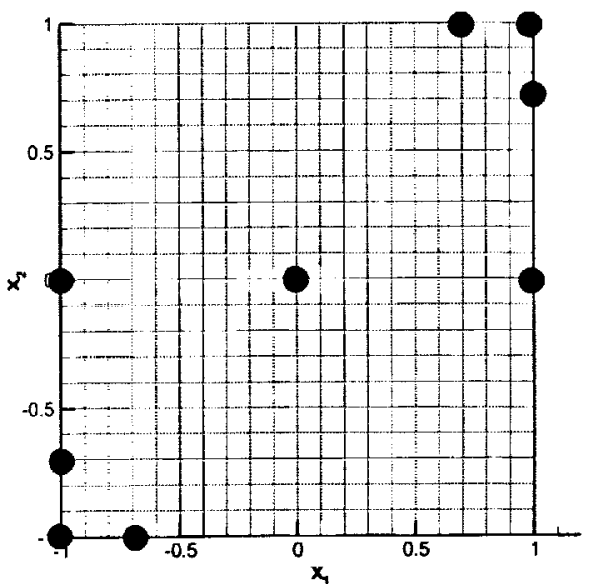

(b) RS 3 (Error-based DSW)

Figure 7: Design space of global RS models for quartic polynomial

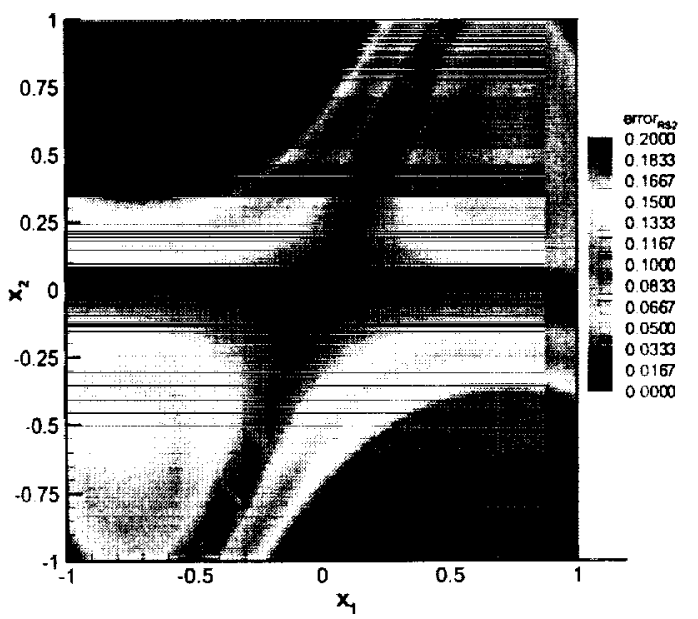

(a) RS 2 (Standard DSW)

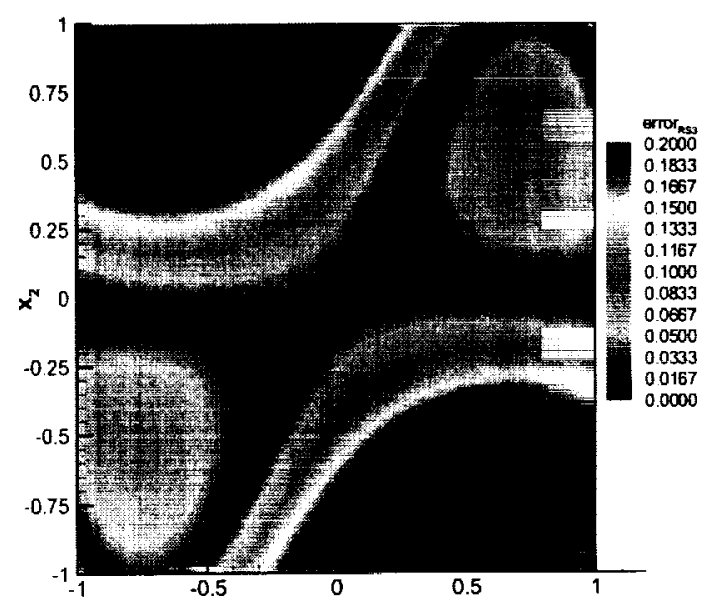

(b) RS 3 (Error-based DSW)

Figure 8: Error contours after DSW in quartic polynomial 


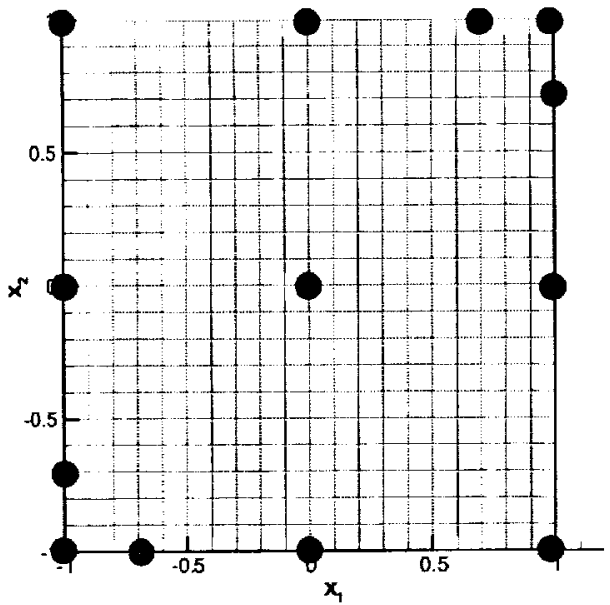

(a) Data points

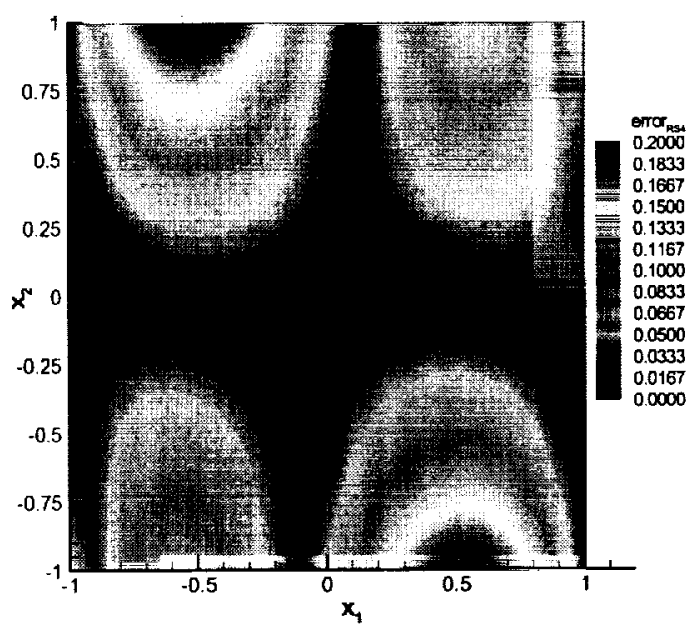

(b) Error contours

Figure 9: RS4 for quartic polynomial (RS 1+RS 3 design points)

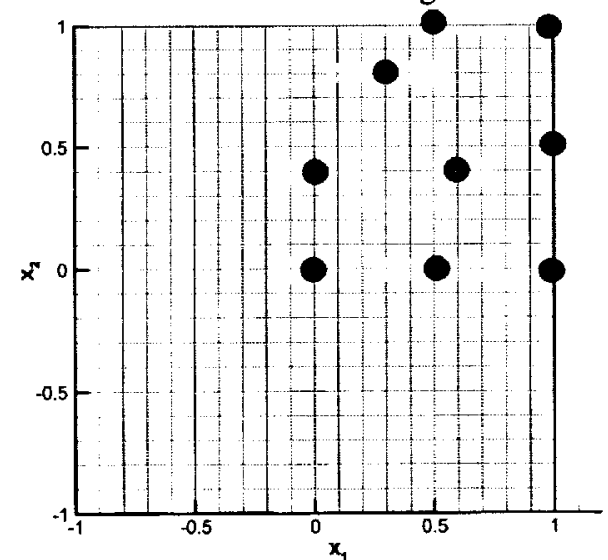

(a) RS 5 (Standard DSW)

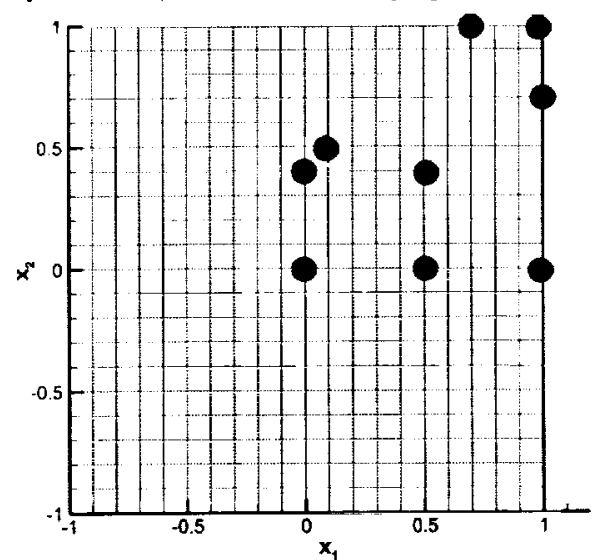

(b) RS 6 (Error-based DSW)

Figure 10: Design space of local RS models for quartic polynomial in upper-right quadrant

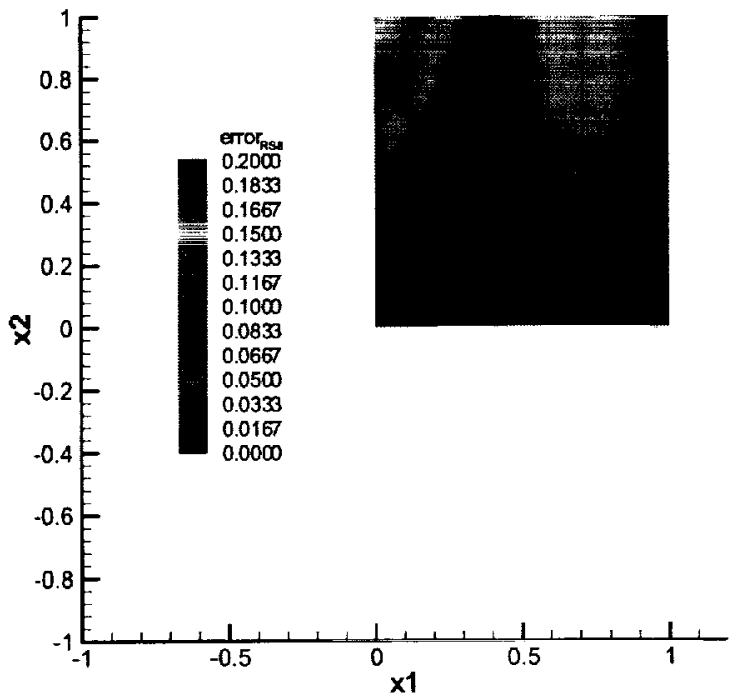

(a) RS 5 (Standard DSW)

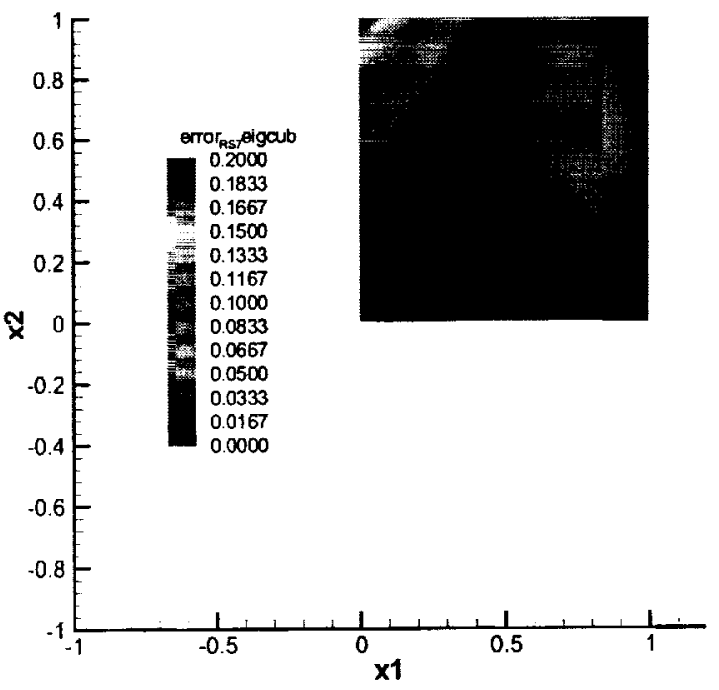

(b) RS 6 (Error-based DSW)

Figure 11: Error contours for quartic polynomial in upper-right quadrant 


\section{APPENDIX}

\section{Response Surface Methodology}

Response surface approximations fit numerical or physical experimental data with an analytical model that is usually a low-order polynomial. The response at design point $\mathrm{x}^{*}$ is denoted as $y[=y(\mathbf{x})]$ and given by Eq. (4)

$$
y(\mathbf{x})=\eta(\mathbf{x})+\varepsilon
$$

where $\eta(\mathbf{x})$ is the true mean response at design point $\mathbf{x}$ and $\varepsilon$ represents other random sources of variation such as measurement error and noise not accounted for in $\eta$. In other words if the experimenter has the luxury of performing the experiment many times at $\mathbf{x}$, the average of the observations will tend to $\eta$ despite the random errors $\varepsilon$. Random errors $\varepsilon$ are assumed to be uncorrelated and normally distributed random variable with zero mean and standard deviation $\sigma$, which is the same at all points. In RS technique the true mean response is assumed to be given in terms of coefficients $\beta_{i}$ s and shape functions $f_{i}(\mathbf{x})\left(i=1, n_{b}\right)$ as in Eq. (5).

$$
\eta(\mathbf{x})=\sum_{i=1}^{n_{b}} \beta_{i} f_{i}(\mathbf{x})=\mathbf{f}^{\mathbf{T}} \mathbf{\beta}
$$

where $\mathbf{f}^{\mathbf{T}}=\left\{f_{1}(\mathbf{x}) \quad f_{2}(\mathbf{x}) \quad \cdots \quad f_{n_{b}}(\mathbf{x})\right\}$ and $\boldsymbol{\beta}=\left\{\begin{array}{llll}\beta_{1} & \beta_{2} & \cdots & \beta_{n_{b}}\end{array}\right\}^{\mathbf{T}}$ and $\mathbf{T}$ denotes transpose for the matrix representation. The $n_{b}$ shape functions $f_{i}$ are usually monomials and $\beta_{i}$ are unknown coefficients, representing the best approximation to $y$ when noise is absent. With noise the fitted approximation is given as

$$
\hat{y}(\mathbf{x})=\sum_{i=1}^{n_{b}} b_{i} f_{i}(\mathbf{x})=\mathbf{f}^{\mathrm{T}} \mathbf{b}
$$

where $b_{i} s$ (vector $\mathbf{b}$ ) are estimates of the $\beta_{i}$ (vector $\boldsymbol{\beta}$ ) obtained from a least squares fit. The difference (residual) between the data $y$ for a point $\mathbf{x}$ and the estimate defined in Eq. (6) is given as

$$
e=y-\hat{y}(\mathbf{x})
$$

The residual for the $N$ data points can now be written in matrix form,

$$
\mathbf{e}=\mathbf{y}-\mathbf{X}_{1} \mathbf{b}_{1}
$$

where $\mathbf{X}_{1}$ is the matrix whose terms in the row associated with the point $\mathbf{x}$ are formed by monomials $f_{i}(\mathbf{x})^{\star}$. For instance, for a quadratic model in two-variables with $N$ data points $\mathbf{X}_{1}$ is given as

$$
\mathbf{X}_{1}=\left[\begin{array}{cccccc}
1 & x_{11} & x_{21} & x_{11}^{2} & x_{11} x_{21} & x_{21}^{2} \\
1 & x_{12} & x_{22} & x_{12}^{2} & x_{12} x_{22} & x_{22}^{2} \\
\vdots & \vdots & \vdots & \vdots & \vdots & \vdots \\
1 & x_{1 j} & x_{2 j} & x_{1 j}^{2} & x_{1 j} x_{2 j} & x_{2 j}^{2} \\
\vdots & \vdots & \vdots & \vdots & \vdots & \vdots \\
1 & x_{1 N} & x_{2 N} & x_{1 N}^{2} & x_{1 N} x_{2 N} & x_{2 N}^{2}
\end{array}\right]
$$

The coefficient vector $\mathbf{b}_{1}$ in Eq. (8) is solved for minimum residual vector in a least-square sense, and can be expressed as

$$
b_{1}=\left(X_{1}^{T} X_{1}\right)^{-1} X_{1}^{T} \mathbf{y}
$$

When the model is exact (no bias error), unbiased estimates of the noise $\sigma$ in the data is given as

$$
s=\sqrt{\frac{\mathbf{y}^{\mathbf{T}} \mathbf{y}-\mathbf{b}_{1}^{\mathbf{T}} \mathbf{X}_{1}^{\mathbf{T}} \mathbf{y}}{N-n_{b}}}
$$

With finite number of data, errors in the data cause errors in the coefficients, and that in turn causes a prediction error of the RS approximation that depends on location. Because Eq. (5) is only an approximation to the true mean

* Bold face is used for vector and matrix representations, e.g. $x=\left[\begin{array}{ll}x_{1} & x_{2}\end{array}\right]^{\mathrm{T}}$ for two-variable case where superscript T stands for transpose operation

" The subscript " 1 " used for $p_{1}, \mathbf{b}_{1}$ and $\mathbf{X}_{1}$ refers to the set of monomials included in the model. Later, a subscript " 2 " will be used for the set of monomials needed to complete the model in order to obtain the true response. 
response function, $s$ will contain not only noise error, but also error due to the approximation. Besides $s$, the quality of the approximation is often measured by the adjusted coefficient of multiple determination $R_{a}^{2}$.

$$
R_{a}^{2}=1-\frac{s^{2}}{\sum_{j=1}^{N}\left(y_{j}-\bar{y}\right)^{2} /(N-1)}
$$

where $\bar{y}$ is the average value of the response data.

\section{D-Optimal Design}

A D-optimal design minimizes the generalized variance of the estimates, which is equivalent to maximizing the determinant of the moment matrix, $\mathbf{M}$ [15].

$$
|\mathbf{M}|=\frac{\left|\mathbf{X}^{\mathbf{T}} \mathbf{X}\right|}{N^{n_{b}}}
$$

The D-optimal design approach makes use of the knowledge of the properties of polynomial model in selecting the design points. This criterion tends to emphasize the terms of the polynomial model with the highest sensitivity [17].

\section{Mean Squared Error Criterion [9]}

As a measure of the error in the approximation, the mean squared error of prediction MSEP defined as in Eq. (14) is used

$$
\operatorname{MSEP}(\mathbf{x})=E[\hat{y}(\mathbf{x})-\eta(\mathbf{x})]^{2}
$$

where $\eta(\mathbf{x})$ and $\hat{y}(\mathbf{x})$ are the true mean response and the prediction by the fitted model, respectively at $\mathbf{x} . M S E P$ is by definition an expected value that would be reached if the number of data used in approximation were unlimited. Equation (14) may be rewritten as

$$
M S E P=E[\hat{y}(\mathbf{x})-E \hat{y}(\mathbf{x})]^{2}+[E \hat{y}(\mathbf{x})-\eta(\mathbf{x})]^{2}
$$

The first term in Eq. (15) represents the variance error due to random noise and the second term represents bias error due to inadequate modeling. This error expression is usually averaged via integration over the design space and the integral is minimized by choosing the experimental designs (DOE) that control the effect of one or both types of error-noise and bias-[18 and 5]. It is reported in [18], "the averaging MSEP over the design region in fact mask a poor performance by the RS approximation at certain locations of the design region". Papila and Haftka [9] instead attempted point-wise characterization of the error and to determine the design regions where RS prediction may suffer due to either or both types of error. Therefore Eq. (15) is used to investigate the variation of MSEP from point-to-point.

The expectation of the predicted response at a given design point $\mathbf{x}$ can be expressed as

$$
E \hat{y}(\mathbf{x})=\mathbf{f}_{1}^{\mathbf{T}} E\left(\mathbf{b}_{1}\right)
$$

where $\mathbf{f}_{1}$ * is the vector of shape functions $f_{i}$ [see Eq. (5)] calculated at point $\mathbf{x}$.

The mean and the variation for the coefficient estimates are given as

$$
\begin{aligned}
& E\left(\mathbf{b}_{1}\right)=\left(\mathbf{X}_{1}^{\mathrm{T}} \mathbf{X}_{1}\right)^{-1} \mathbf{X}_{1}^{\mathrm{T}} E(\mathbf{y}) \\
& \operatorname{Var}\left(\mathbf{b}_{1}\right)=\sigma^{2}\left(\mathbf{x}_{1}^{\mathrm{T}} \mathbf{X}_{1}\right)^{-1}
\end{aligned}
$$

where $\sigma$ is the standard deviation of the noise error. The first part of the mean squared error in Eq. (15) is equal to the prediction variance at $\mathbf{x}$.

$$
\begin{aligned}
E[\hat{y}(\mathbf{x})-E \hat{y}(\mathbf{x})]^{2} & =\operatorname{Var}[\hat{y}(\mathbf{x})] \\
& =\mathbf{f}_{1}^{\mathrm{T}} \operatorname{Var}\left(\mathbf{b}_{\mathbf{1}}\right) \mathbf{f}_{\mathbf{1}} \\
& =\sigma^{2} \mathbf{f}_{1}^{\mathrm{T}}\left(\mathbf{x}_{1}^{\mathrm{T}} \mathbf{X}_{1}\right)^{-1} \mathbf{f}_{\mathbf{1}}
\end{aligned}
$$

The second part of Eq. (15) is the squared error due to the inadequacy of the model used

* Subscripts 1 and 2 assign matrices and vectors for the fitting model and the missing terms, respectively. 
AIAA 2002-0539

$$
[E \hat{y}(\mathbf{x})-\eta(\mathbf{x})]^{2}=(\operatorname{Bias}[\hat{y}(\mathbf{x})])^{2}
$$

Therefore mean squared error of prediction in Eq. (15) can be rewritten as

$$
\operatorname{MSEP}=\operatorname{Var}[\hat{y}(\mathbf{x})]+(\operatorname{Bias}[\hat{y}(\mathbf{x})])^{2}
$$

The form of the prediction variance, $\operatorname{Var}[\hat{y}(\mathbf{x})]$, in Eq (19) does not change even in the presence of inadequate modeling, since variance is only caused by random noise. However, when bias error is present, the residual error mean square $s^{2}$ is not an unbiased estimate of $\sigma^{2}$. Using Eqs. (10) and (11) we get

$$
s^{2}=\frac{\mathbf{y}^{\mathrm{T}}\left(\mathbf{I}_{\mathbf{N}}-\mathbf{P}\right) \mathbf{y}}{N-n_{b}}
$$

where $\mathbf{P}=\mathbf{X}_{1}\left(\mathbf{X}_{1}^{\mathrm{T}} \mathbf{X}_{1}\right)^{-1} \mathbf{X}_{1}^{\mathrm{T}}$

The accuracy of this estimate depends on factors such as the available data or number of points and also the adequacy of the fitting model that determines whether the estimate is unbiased or biased.

The true mean response at $\mathbf{x}$ can be written as

$$
\eta(\mathbf{x})=\mathbf{f}_{1}^{\mathrm{T}} \boldsymbol{\beta}_{1}+\mathbf{f}_{2}^{\mathrm{T}} \boldsymbol{\beta}_{2}
$$

where $\mathbf{f}_{2}$ are terms missing from the assumed model. Since one usually does not know the true response, it is often assumed to be a higher order polynomial when monomials are used as shape functions. For instance, for a quadratic fitting model in two-variables when true function is cubic

$$
\begin{aligned}
& \mathbf{f}_{1}^{\mathbf{T}}=\left[\begin{array}{llllll}
1 & x_{1} & x_{2} & x_{1}^{2} & x_{1} x_{2} & x_{2}^{2}
\end{array}\right] \\
& \boldsymbol{\beta}_{1}^{\mathbf{T}}=\left[\begin{array}{llllll}
\beta_{11} & \beta_{12} & \beta_{13} & \beta_{14} & \beta_{15} & \beta_{16}
\end{array}\right] \\
& \mathbf{f}_{2}^{\mathrm{T}}=\left[\begin{array}{llll}
x_{1}^{3} & x_{1}^{2} x_{2} & x_{1} x_{2}^{2} & x_{2}^{3}
\end{array}\right] \\
& \boldsymbol{\beta}_{2}=\left[\begin{array}{llll}
\beta_{21} & \beta_{22} & \beta_{22} & \beta_{22}
\end{array}\right] \\
& E(\mathbf{y})=\mathbf{X}_{1} \boldsymbol{\beta}_{1}+\mathbf{X}_{2} \boldsymbol{\beta}_{2}
\end{aligned}
$$

where $\mathbf{X}_{2}$ is similar to $\mathbf{X}_{1}$, but due to the terms (shape functions) present in the true response that are not included in the fitting model. After substitution of Eq. (26), Eq. (17) becomes

$$
E\left(\mathbf{b}_{1}\right)=\boldsymbol{\beta}_{1}+\mathbf{A} \boldsymbol{\beta}_{2}
$$

where $A=\left(X_{1}^{T} X_{1}\right)^{-1} X_{1}^{T} X_{2}$ is called the alias matrix. Substitution of Eq. (27) into Eq. (16) yields

$$
\begin{aligned}
& E \hat{y}(\mathbf{x})=\mathbf{f}_{1}^{\mathbf{T}}\left(\boldsymbol{\beta}_{\mathbf{1}}+\mathbf{A} \boldsymbol{\beta}_{\mathbf{2}}\right) \\
& \begin{aligned}
(B \operatorname{Bias}[\hat{y}(\mathbf{x})])^{2}= & \left\{\mathbf{f}_{1}^{\mathbf{T}}\left(\boldsymbol{\beta}_{\mathbf{1}}+\mathbf{A} \boldsymbol{\beta}_{\mathbf{2}}\right)-\mathbf{f}_{1}^{\mathbf{T}} \boldsymbol{\beta}_{\mathbf{1}}-\mathbf{f}_{2}^{\mathbf{T}} \boldsymbol{\beta}_{\mathbf{2}}\right\} \\
& =\boldsymbol{\beta}_{2}^{\mathbf{T}}\left[\mathbf{A}^{\mathbf{T}} \mathbf{f}_{\mathbf{1}}-\mathbf{f}_{\mathbf{2}}\right]\left[\mathbf{f}_{1}^{\mathrm{T}} \mathbf{A}-\mathbf{f}_{2}^{\mathbf{T}} \mid \boldsymbol{\beta}_{\mathbf{2}}\right.
\end{aligned}
\end{aligned}
$$

The MSEP at a given point can now be estimated as by using $s^{2}$ instead of $\sigma^{2}$

$$
\begin{aligned}
& M \hat{S E} P(\mathbf{x})=s^{2} \mathbf{f}_{1}^{\mathbf{T}}\left(\mathbf{X}_{\mathbf{1}}^{\mathrm{T}} \mathbf{X}_{\mathbf{1}}\right)^{-\mathbf{1}} \mathbf{f}_{\mathbf{1}}+\boldsymbol{\beta}_{2}^{\mathbf{T}} \mathbf{M} \boldsymbol{\beta}_{\mathbf{2}} \\
& \text { where } \mathbf{M}=\left[\mathbf{A}^{\mathbf{T}} \mathbf{f}_{\mathbf{1}}-\mathbf{f}_{\mathbf{2}}\right]\left[\mathbf{f}_{\mathbf{1}}^{\mathrm{T}} \mathbf{A}-\mathbf{f}_{\mathbf{2}}^{\mathbf{T}}\right]
\end{aligned}
$$

Note that MSEP is an expectation by definition [Eq. (14)], and Eq. (30) is its estimate by the data available and associated $s^{2}$. Using $E(\mathbf{y})$ from Eq. (26), the expected value of biased error mean square $s^{2}$ given by Seber [19]

$$
E\left(s^{2}\right)=\sigma^{2}+\frac{\boldsymbol{\beta}_{2}^{\mathbf{T}} \mathbf{X}_{2}^{\mathbf{T}}\left(\mathbf{I}_{\mathbf{N}}-\mathbf{P}\right) \mathbf{X}_{2} \boldsymbol{\beta}_{\mathbf{2}}}{N-p_{1}}
$$

since $\left(\mathbf{I}_{\mathbf{N}}-\mathbf{P}\right)$ is an idempotent matrix, that is a matrix whose square is equal to itself. It is also positive semidefinite matrix, so $E\left(s^{2}\right) \geq \sigma^{2}$ provided that $\left(\mathbf{I}_{N}-\mathbf{P}\right) \mathbf{X}_{2} \boldsymbol{\beta}_{2} \neq 0$. Equation (32) means that when bias errors are present, $s^{2}$ is a biased estimate of $\sigma^{2}$.

If this expected value, $E\left(s^{2}\right)$, is substituted in prediction variance contribution in Eq. (30) expected value for the estimate $M \hat{S E P}$ can be expressed as 


$$
\begin{aligned}
& E[M \hat{S} E P(\mathbf{x})] \cong \mathbf{f}_{1}^{\mathbf{T}}\left(\mathbf{X}_{1}^{\mathbf{T}} \mathbf{X}_{1}\right)^{-\mathbf{1}} \mathbf{f}_{\mathbf{1}}\left[\sigma^{2}+\frac{\boldsymbol{\beta}_{2}^{\mathbf{T}} \mathbf{K} \boldsymbol{\beta}_{2}}{N-p_{1}}\right]+\boldsymbol{\beta}_{2}^{\mathbf{T}} \mathbf{M} \boldsymbol{\beta}_{2} \\
& \equiv \sigma^{2} \mathbf{f}_{1}^{\mathrm{T}}\left(\mathbf{X}_{1}^{\mathrm{T}} \mathbf{X}_{1}\right)^{-1} \mathbf{f}_{1}+\boldsymbol{\beta}_{2}^{\mathrm{T}} \mathbf{G} \boldsymbol{\beta}_{2} \\
& \text { where } \mathbf{K}=\mathbf{X}_{2}^{\mathrm{T}}\left(\mathbf{X}_{\mathbf{2}}-\mathbf{X}_{1} \mathrm{~A}\right) \\
& \mathbf{G}=\frac{\mathbf{f}_{\mathbf{1}}^{\mathrm{T}}\left(\mathbf{X}_{\mathbf{1}}^{\mathrm{T}} \mathbf{X}_{\mathbf{1}}\right)^{-\mathbf{1}} \mathbf{f}_{\mathbf{1}}}{N-p_{1}} \mathbf{K}+\mathbf{M}
\end{aligned}
$$

The aim of the method is to identify points where the bias error is large. It is assumed that the terms missing from the fitting model are known, but there is not enough data to calculate the corresponding coefficients $\boldsymbol{\beta}_{\mathbf{2}}$. If one can estimate the size of $\beta_{2}$, it is possible to formulate a constrained maximization problem for the largest magnitude of the mean squared error predictor that may be experienced at any given design point for the worst possible $\boldsymbol{\beta}_{2}$ of that magnitude.

$$
\begin{aligned}
& \max _{\boldsymbol{\beta}_{2}} E[M \hat{S} E P(\mathbf{x})] \\
& \text { such that }\left\|\boldsymbol{\beta}_{\mathbf{2}}\right\|^{2}=c
\end{aligned}
$$

The Lagrangian for this optimization problem can be written as

$$
L\left(\boldsymbol{\beta}_{2}, \lambda\right)=E[M \hat{S} E P(\mathbf{x})]+\lambda\left(\boldsymbol{\beta}_{2}^{\mathbf{T}} \boldsymbol{\beta}_{2}-c\right)
$$

Differentiating the Lagrangian with respect to $\boldsymbol{\beta}_{2}$

$$
\nabla\left[\sigma^{2} \mathbf{f}_{1}^{\mathrm{T}}\left(\mathbf{X}_{1}^{\mathrm{T}} \mathbf{X}_{1}\right)^{-1} \mathbf{f}_{1}\right]+\nabla\left(\boldsymbol{\beta}_{2}^{\mathrm{T}} \mathbf{G} \boldsymbol{\beta}_{2}\right)+\lambda \nabla\left(\boldsymbol{\beta}_{2}^{\mathrm{T}} \boldsymbol{\beta}_{2}-c\right)=0
$$

where $\nabla=\left[\begin{array}{llll}\frac{\partial}{\partial \beta_{21}} & \frac{\partial}{\partial \beta_{22}} & \cdots & \frac{\partial}{\partial \beta_{2 p_{2}}}\end{array}\right]^{T}$. Equation (38) yields the following eigenvalue problem at a design point $\mathbf{x}_{j}$

$$
\begin{aligned}
& \mathbf{G}_{2}+\lambda \boldsymbol{\beta}_{2}=\mathbf{0} \text { or } \\
& \mathbf{G} \boldsymbol{\beta}_{2}-\lambda_{G} \boldsymbol{\beta}_{2}=\mathbf{0}
\end{aligned}
$$

for which the maximum eigenvalue $\lambda_{G \max }$ characterizes the maximum possible mean squared and bias error associated with the assumed true model that includes shape functions missing in the fitting model. The corresponding eigenvector defines the coefficients of the missing shape functions that results in the largest bias error when fitted only with the assumed model. The eigenvectors and the function experiencing the worst possible bias error may be different point-to-point although the magnitude of the missing coefficient vector is constrained. So the eigenvalue calculated does not reflect the true function corresponding to the data (as the data is insufficient to calculate $\boldsymbol{\beta}_{2}$ ). It reflects instead, the assumed form of true function with the shape function coefficients $\boldsymbol{\beta}_{\mathbf{2}}$ (among all the possible combinations such that $\boldsymbol{\beta}_{2}^{\mathrm{T}} \boldsymbol{\beta}_{2}=c$ ) causing the largest error.

As can be seen from the derivation the eigenvalue error measure strongly depends on the DOE used, but not on the response data. This independence from the response data may be misleading especially for the cases where fitting model predicts the true function very well at the data points. The following $2 \mathrm{D}$ cubic polynomial example demonstrates such a case.

$$
y=1+x_{1}^{3}+x_{2}^{3}
$$

Face-centered central composite design (FCCD) in 2D was used for constructing a quadratic RS. Figure 1 presents the FCCD points and relevant $\sqrt{\lambda_{G}}$ field. The maximum values are at the data points on the boundary, but Table A1 indicates a perfect with the nine data points. The testing rms-error (using 21 by 21 grid points except the nine data points) and the maximum error, however, shows that the fit is actually quite poor. Figure Al presents the true function and the error field. Comparing Figure Alb and Figure $\mathrm{lb}$ indicates that eigenvalue error measure did not predict the high-error regions in this particular example, unlike for the examples studied and reported in [9]. It is expected that the benefit of the error measure will be increased further if the response data can also be used in the derivation. 
Table A1: Statistics of the quadratic RS for cubic polynomial given in Eq. (40). RS is given is $\hat{y}=1+x_{1}+x_{2}$

\begin{tabular}{|l|c|}
\hline RSquare & 1.000 \\
\hline RSquare Adj & 1.000 \\
\hline rms-error Predictor & 0.000 \\
\hline Mean & 1.000 \\
\hline Observations, $N$ & 9 \\
\hline Testing rms-error (21 x 21 grid) & 0.385 \\
\hline Max. Error & 0.768 \\
\hline
\end{tabular}

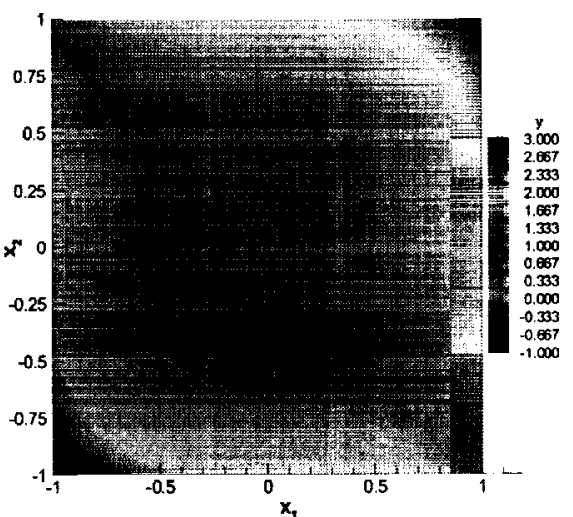

(a) True function

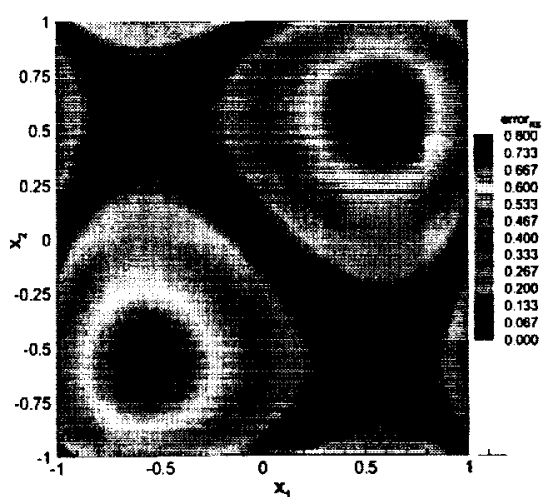

(b) Absolute error of RS1

Figure Al: Contours and RS errors for cubic problem in Eq. (40) 